\title{
A Responsive Design Approach for Supporting Mobile Access to Virtual and Remote Laboratories
}

\author{
Panagiotis Zervas ${ }^{1,2}$, Alexandros Trichos ${ }^{1}$, Demetrios G Sampson ${ }^{1,2}, \mathrm{Na} \mathrm{Li}^{3}$ \\ ${ }^{1}$ Department of Digital Systems \\ University of Piraeus \\ Piraeus, Greece \\ ${ }^{2}$ Information Technologies Institute \\ Centre for Research and \\ Technology Hellas, Thessaloniki, \\ Greece \\ ${ }^{3}$ École Polytechnique Fédérale de \\ Lausanne (EPFL), Lausanne, \\ Switzerland \\ e-mail: pzervas@iti.gr, alexandros.trichos@iti.gr, sampson@iti.gr, na.li@epfl.ch
}

\begin{abstract}
Remote and virtual laboratories aim to overcome typical constraints of traditional laboratories and offer access to laboratory experiments without location and time restrictions. Additionally, the continuous growth of mobile devices' ownership, as well as their increased technical capabilities, have created an emerging trend for supporting mobile access to virtual and remote labs. Existing approaches in this direction are focusing on developing device-dependent mobile apps for accessing the experiment(s) of the original remote or virtual lab. This creates difficulties related to (a) seamless access to experiments of remote or virtual labs across various mobile devices and (b) additional costs that are needed for developing different versions of the same mobile app that meets the specific technical requirements of each mobile device. In this paper, we aim to address this issue by adopting a responsive design approach for developing mobile web apps towards providing seamless access through various mobile devices to existing virtual and remote labs.
\end{abstract}

Keywords-Virtual labs; remote labs; mobile access; responsive design; science education

\section{INTRODUCTION}

Remote and virtual laboratories are increasingly prevalent alternatives to the face to face traditional laboratories [1], [2]. Remote labs are defined as "computer-controlled laboratories, which can be accessed and controlled externally through some communication medium" [3]. On the other hand, virtual labs are defined as: "interactive environments for designing and conducting simulated experiments" [4]. Both remote and virtual labs can facilitate students to conduct experiments outside the formal borders of classroom teaching. Moreover, it has been recognized that these environments can significantly enhance students' interest in science and develop their inquiry skills [5], [6].

At the same time, mobile devices have been investigated as an alternative medium for providing access to virtual and remote labs [7], [8].To this end, there are a number of works in the literature that deal with this issue [7], [8], [9], [10]. By reviewing these works, we can identify that all of them are focusing on developing device-dependent mobile apps for accessing the experiment(s) of the original remote or virtual lab. The limitations of this approach are that (a) devicedependent mobile apps can not offer seamless access to various experiments of remote or virtual labs through different mobile devices and (b) additional development costs are required for developing different versions of the same mobile app in order to accommodate the diverse types of mobile devices available [11]. In this paper, we aim to address this issue by adopting a responsive design approach for developing mobile web apps towards providing access across various mobile devices to existing virtual and remote labs.

\section{MOBILE DEVELOPMENT APPROACHES}

Currently, there are three main approaches for developing mobile apps, which can be summarized as follows [11]: (a) Native Mobile Apps: these apps are specifically designed to work on the operating system of a specific mobile device (such as Android, iOS, Windows Phone, Blackberry 10 or Nokia's S40). Native mobile apps are programmed using specific programming languages that are supported by development frameworks, which are provided by operating systems' vendors. Typically, native mobile apps installed on the mobile device, but the user data can be stored either on the mobile device and/or in the cloud (remotely), (b) Mobile Web Apps: these web apps are designed to run on a mobile device and they are also referred to as mobile rich internet applications (RIA). Both mobile web apps and their user data are stored in the cloud, and (c) Hybrid Mobile Apps: they support multiple operating systems by packaging the content as a native mobile app to be installed directly to the mobile device, enabling the combination of native mobile apps' advantages with crossplatform interoperability.

Native and hybrid mobile apps are usually available via mobile apps' distribution platforms, which are referred to as mobile app markets or mobile app stores [11]. However, this implies that these types of mobile apps (a) should undergo the lengthy review process of mobile app stores before becoming available to their end-users and (b) end-users should download the install them to their devices. On the other hand, mobile web apps are browser-based (there is no need for downloading) and they do not need to be distributed via mobile app stores. They have also the advantage to be platform and device independent, when they are designed and developed by following specific approaches. Such approaches for designing and developing mobile web apps are the following [12]:

- Adaptive Design: it uses the server to detect the device the mobile web app is being viewed on. It is based on templates for each device type. This means that it calls up a dedicated HTML5 / CSS set for each device. 
- Responsive Design: it uses specific CSS code (namely, CSS media queries) in order to detect the current device and modify the presentation of the mobile web app accordingly. It is based on a single HTML5 / CSS set, which is in turn fluidly adjusted to fit any device.

As we can notice, adaptive design ensures that a mobile web app will change to fit a predetermined set of screen and device sizes. This implies development and maintenance of separate HTML/CSS sets for predetermined screen and device sizes. On the other hand, responsive design ensures that the mobile web app will fluidly change and respond to fit any screen or device size based on a single HTML / CSS set. As a result, we have adopted a responsive design approach for developing web apps to support seamless access to existing virtual and remote labs is across any mobile device. This approach could also eliminate the costs for the development and maintenance of separate HTML/CSS sets for various mobile devices.

\section{RESPONSIVE DESIGN FRAMEWORKS}

In this section, we provide an overview of existing responsive design frameworks that facilitate the process of developing responsive mobile web apps. More specifically, we have identified four (4) responsive design frameworks, namely: (a) Twitter Bootstrap ${ }^{1}$, which has been developed by Twitter developers, (b) Foundation ${ }^{2}$ that has been developed by Zurb (a US software company), (c) Skeleton ${ }^{3}$ and (d) HTML5 Boilerplate ${ }^{4}$. The latter two have been developed by communities of independent developers. Table I summarizes the features of the indentified responsive design frameworks.

TABLE I. COMPARING EXISTING RESPONSIVE DESIGN FRAMEWORKS

\begin{tabular}{|c|c|c|c|c|}
\hline Features & $\begin{array}{c}\text { Twitter } \\
\text { Bootstrap }\end{array}$ & Foundation & Skeleton & $\begin{array}{c}\text { HTML5 } \\
\text { Boilerplate }\end{array}$ \\
\hline $\begin{array}{c}\text { HTML/CSS } \\
\text { Templates }\end{array}$ & $\checkmark$ & $\checkmark$ & $\checkmark$ & $\checkmark$ \\
\hline $\begin{array}{c}\text { Scripts / } \\
\text { Utilities }\end{array}$ & $\checkmark$ & $\checkmark$ & - & - \\
\hline UI Elements & $\checkmark$ & $\checkmark$ & Limited & Limited \\
\hline Widgets & $\checkmark$ & $\checkmark$ & - & - \\
\hline Documentation & $\checkmark$ & Limited & $\checkmark$ & $\checkmark$ \\
\hline $\begin{array}{c}\text { Community } \\
\text { Support }\end{array}$ & $\checkmark$ & Limited & $\checkmark$ & $\checkmark$ \\
\hline
\end{tabular}

As we can notice from Table I, all existing frameworks offer basic HTML and CSS templates for designing and developing a mobile web app but only two, namely Twitter Bootstrap and Foundation provide a complete set of scripts/utilities, UI elements and widgets. More specifically, these features provide developers with script libraries that can be utilized for layout optimization, as well as with standard and more complex UI elements that can be used for enhancing user experience across devices. Additionally, a strong point in favour of Twitter Bootstrap is the large community support in contrast to the limited documentation and support of Foundation. For the aforementioned reasons, we considered that the most suitable option was to select Twitter Bootstrap framework for developing responsive web apps for providing access to existing virtual and remote labs.

\section{A CASE StUdy OF APPLYING THE PROPOSED RESPONSIVE DESIGN APPROACH}

In this section, we present the application of our proposed approach in the framework of a major European Initiative namely, the "Go-Lab: Global Online Science Labs for Inquiry Learning at School". The Go-Lab Project (http://www.go-lab-project.eu/) aims to establish an online portal that will facilitate the federation of existing virtual and remote labs. The Go-Lab Portal will enable lab owners to promote their labs and K12 teachers to discover and use virtual and remote labs for designing pedagogically engaging and motivating learning activities for their students [13].

Within this context, we aim to support students to execute across different devices the learning activities that are designed by their teachers based on existing virtual and remote labs. To this end, we have applied the proposed responsive design approach towards providing students with the same user experience across devices when they access existing virtual and remote labs. More specifically, we have applied the following main responsive design principles:

- Media queries, which optimize the design of the web app for different viewing contexts and spontaneously fix any inconsistencies that occur at different resolution ranges.

- Fluid layout which ensures that the web app can scale to a browser's full width.

- Flexible media, which means that images and videos can work in a flexible context and resize according to different resolution ranges.

Fig.1 presents the desktop version (http://tinyurl.com/ilsgolab) of a web app that provides access to an existing virtual lab, whereas Fig. 2 presents the mobile version of the same web app by utilizing the aforementioned responsive design principles.

As we can notice from Fig. 1 and Fig. 2, the desktop version of the web app, having enough width to unwrap its content, displays both text and media in the originally intended sizes. On a mobile device, the available width is considerably reduced. The responsive design approach changes fluidly the content of the web app to fit the new screen size. This means that the new layout wraps the text and enlarges the images and videos in a responsive way that augments readability.

\footnotetext{
${ }^{1}$ http://getbootstrap.com/

${ }^{2}$ http://foundation.zurb.com/

${ }_{3} \mathrm{http://www.getskeleton.com} /$

${ }^{4} \mathrm{http} / / \mathrm{htm} 15$ boilerplate.com/
} 


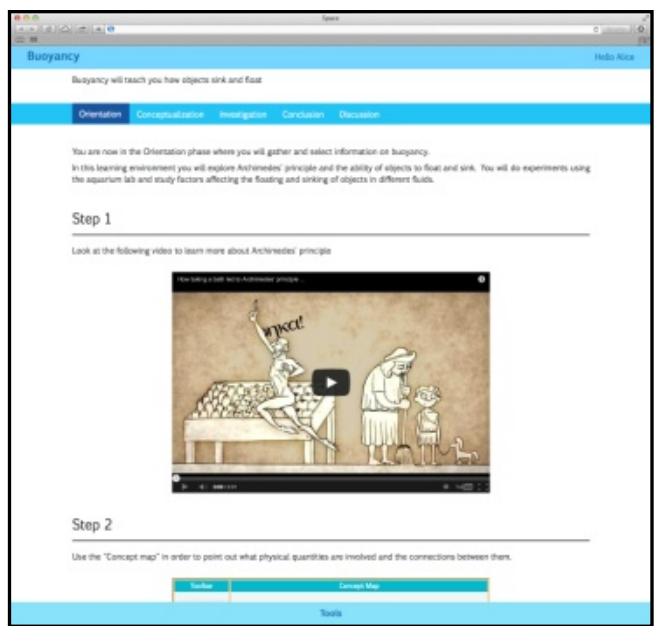

Figure 1. Desktop Version of a web app for accessing a virtual lab

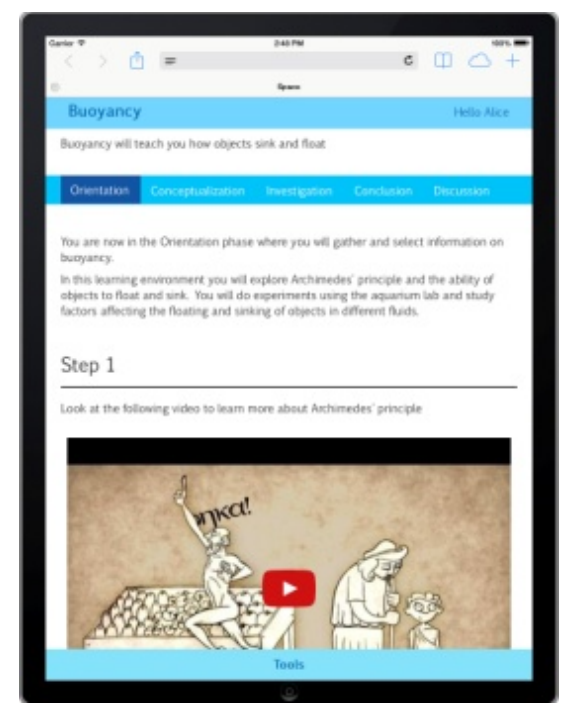

Figure 2. Mobile Version of the web app for accessing the same virtual lab

\section{CONCLUSIONS AND FUTURE WORK}

In this paper, it was argued that there is an increased interest for supporting mobile access existing virtual and remote labs. However, existing approaches in this direction are focusing on developing device-dependent mobile apps. As a result, a responsive design approach was proposed for supporting mobile access across devices to existing virtual and remote labs. This approach is applied in the framework of a major European Initiative namely, the "Go-Lab: Global Online Science Labs for Inquiry Learning at School" to create the essential technical infrastructure for enabling seamless access to various experiments of remote and virtual labs through different mobile devices. This approach is expected to enable broader reach of students for accessing the various experiments of remote and virtual labs offered by the Go-Lab Project without the need for extending the current technical infrastructure.

Future work will include measurement of the possible impact that can be achieved with the proposed approach in terms of students' engagement to various experiments of remote and virtual labs offered by the Go-Lab Project.

\section{ACKNOWLEDGMENTS}

The work presented in this paper has been partially funded by the European Union in the context of the Go-Lab project (Grant Agreement no. 317601) under the Information and Communication Technologies (ICT) theme of the 7th Framework Programme for R\&D (FP7).

\section{REFERENCES}

[1] T. de Jong, M. C. Linn, and Z. C. Zacharia, "Physical and virtual laboratories in science and engineering education," Science, vol. 340 no. 6130, pp. 305-308, April 2013.

[2] B. Balamuralithara, and P. C. Woods, "Virtual laboratories in engineering education: The simulation lab and remote lab," Computer Applications in Engineering Education, vol. 17 no. 1, pp. 108-118, March 2009.

[3] C. Gravier, J. Fayolle, B. Bayard, M. Ates and J. Lardon, "State of the Art About Remote Laboratories Paradigms - Foundations of Ongoing Mutations," International Journal of Online Engineering, vol. 4, no. 1, September 2008.

[4] L. Gomes and S. Bogosyan, "Current trends in remote laboratories," IEEE Transactions on Industrial Electronics, vol. 56 no. 12, pp. 4744 4756, December 2009.

[5] T. Jaakkola, S. Nurmi, and K. Veermans, "A comparison of students' conceptual understanding of electric circuits in simulation only and simulation-laboratory contexts," Journal of Research in Science Teaching, vol. 48, no. 1, pp. 71-93, January 2011.

[6] T. de Jong, "Technology supports for acquiring inquiry skills," In International encyclopedia of education, B. McGaw, E. Baker and P. Peterson, Eds. Oxford: Elsevier, 2010, pp. 167-171.

[7] P. Orduña, J. García-Zubia, J. Irurzun, D. López-de-Ipiña, and L. Rodriguez-Gil, "Enabling mobile access to Remote Laboratories," Proc. of the IEEE Global Engineering Education Conference (EDUCON) on Learning Environments and Ecosystems in Engineering Education, April, 2011

[8] J. García-Zubía, D. López-de-Ipiña, P. Orduña, "Mobile Devices and Remote Labs in Engineering Education," Proc. of the Eighth IEEE International Conference on Advanced Learning Technologies (ICALT 2008), IEEE Computer Society Press, July 2008, pp. 620622.

[9] A. Al-Zoubi, S. Jeschke, J. Nsour, O. Pfeiffer and T. Richter, "An Electronic Engineering Mobile Remote Laboratory," Proc. of the 8th World Conference on Mobile and Contextual Learning (mLearn 2009), October 2009, pp. 42-48.

[10] D. Cmuk, T. Mutapcic, and M. Borsic, "Mobile Measurement Support for Remote Laboratories and E-Learning Systems," Proc. of the 16th IMEKO TC4 Symposium Exploring New Frontiers of Instrumentation and Methods for Electrical and Electronic Measurements, September 2008.

[11] A. Charland, and B. Leroux, "Mobile application development: web vs. native," Communications of the ACM, vol. 54 no. 5 , pp. 49-53, April, 2011.

[12] S. Amatya, and A. Kurti, "Cross-Platform Mobile Development: Challenges and Opportunities," In Proc. of the Sixth International Conference ICT Innovations 2013, Springer International Publishing, 2014, pp. 219-229.

[13] D. Gillet, T. de Jong, S. Sotiriou, C. Salzmann, "Personalised Learning Spaces and Federated Online Labs for STEM Education at School: Supporting Teacher Communities and Inquiry Learning," Proc. of the of the 4th IEEE Global Engineering Education Conference (EDUCON), March, 2013, 